\title{
FRECUENCIA DE HIPONATREMIA EN PACIENTES CON GASTROENTERITIS AGUDA TRATADA CON LÍQUIDOS INTRAVENOSOS EN EL HOSPITAL DEL NIÑO DR. JOSÉ RENÁN ESQUIVEL ENERO A DICIEMBRE 2016
}

\author{
FREQUENCY OF HYPONATREMIA IN PATIENTS WITH ACUTE GASTROENTERITIS \\ TREATED WITH INTRAVENOUS LIQUIDS IN HOSPITAL DEL NIÑO DR. JOSÉ RENÁN \\ ESQUIVEL JANUARY TO DECEMBER 2016
}

\author{
Alvarado, Antonio*; Gonzales, Héctor*; Alvarado, Manuelt; Tello Montserrat¥ \\ * Médico Residente del Servicio de Pediatría, Hospital Del Niño Dr. José Renán Esquivel, Ciudad de Panamá \\ †Médico Especialista Pediatra Intensivista, Hospital Del Niño Dr. José Renán Esquivel, Ciudad de Panamá \\ ¥Médico Especialista Pediatra Epidemióloga, Hospital Del Niño Dr. José Renán Esquivel, Ciudad de Panamá
}

Recibido: 16 de enero del 2019

Aceptado: 26 de junio del 2020

Autor. Frecuencia de Hiponatremia en Pacientes con Gastroenteritis Aguda Tratada con Líquidos Intravenosos en el Hospital del Niño Dr. José Renán Esquivel Enero a Diciembre 2016. Rev Méd Cient. 2020;33(1):18-25. DOI: 10.37416/rmc.v33i1.513

\section{RESUMEN}

INTRODUCCIÓN: La gastroenteritis aguda es una de las principales causas de morbilidad y mortalidad en la infancia. Numerosos estudios han confirmado la seguridad y eficacia de la terapia de rehidratación oral, sin embargo, la rehidratación intravenosa se continúa utilizando en escenarios donde no es posible hidratar al paciente por vía enteral. Durante la última década, se han acumulado pruebas que sugieren que la administración de un líquido de mantenimiento intravenoso hipotónico en niños hospitalizados, aumenta el riesgo de hiponatremia.

OBJETIVOS GENERALES: Determinar la frecuencia de hiponatremia en pacientes con gastroenteritis que reciben solución salina hipotónica en las salas Pediatría del Hospital del Niño Doctor José Renán Esquivel entre enero a diciembre del 2016.

MATERIALES Y MÉTODOS: Se realizó un observacional de cohorte retrospectivo, descriptivo. Se examinaron los expedientes clínicos de niños sin antecedentes patológicos previos de 1 mes a 15 años, que habían ingresado a sala de pediatría del Hospital del Niño Doctor José Renán Esquivel de enero a diciembre de 2016 con diagnóstico de gastroenteritis aguda. Para la obtención de la muestra se utilizó un $95 \%$ de confianza, la muestra obtenida fue de 190 pacientes y fue calculada utilizando el programa Epi Info versión 7.0

RESULTADOS: No hubo diferencias significativas en el desarrollo de hiponatremia secundario al uso de soluciones salinas hipotónicas

CONCLUSIÓN: No se registró hiponatremia en pacientes con gastroenteritis aguda que reciben soluciones salinas hipotónicas en salas de Pediatría del Hospital del Niño Doctor José Renán Esquivel.

PALABRAS CLAVE: Solución salina hipotónica, gastroenteritis aguda, hiponatremia, convulsiones.

\section{ABSTRACT}

INTRODUCTION: Acute gastroenteritis is one of the main causes of morbidity and mortality in childhood. Numerous studies have confirmed the safety and efficacy of oral rehydration therapy, however, intravenous rehydration is still used in scenarios where it is not possible to hydrate the patient through the enteral route. Over the past decade, evidence has accumulated suggesting that administration of a hypotonic intravenous maintenance fluid in hospitalized children increases the risk of hyponatremia.

GENERAL OBJECTIVE: Determine the frequency of hyponatremia in patients with gastroenteritis receiving hypotonic saline solutions in the Hospital del Niño Doctor José Renán Esquivel between January and December 2016.

MATERIALS AND METHODS: We will carry out a retrospective, descriptive study. To examine the clinical history of children with no previous medical history from 1 month to 15 years who entered the Doctor José Renán Esquivel Children's Hospital pediatric ward from January to December 2016, with a diagnosis of acute gastroenteritis. To obtain the sample we used $95 \%$ confidence, the sample was obtained from 190 patients and was calculated using the Epi Info version 7.0 program

RESULTS: There are no significant differences in the development of hyponatremia to the use of hypotonic saline solutions.

CONCLUSION: There is no record of hyponatremia in patients with acute gastroenteritis who receive hypotonic saline solutions in the Doctor José Renán Esquivel Children's Hospital pediatric ward.

KEYWORDS: Hypotonic solutions, gastroenteritis, hyponatremia, seizures.

FRECUENCIA DE HIPONATREMIA EN PACIENTES CON GASTROENTERITIS AGUDA TRATADA CON LÍQUIDOS INTRAVENOSOS EN EL HOSPITAL DEL NIÑO DR. JOSÉ RENÁN ESQUIVEL ENERO A DICIEMBRE 2016 by Alvarado, Gonzalez, Alvarado, Tello is licensed under a Creative Commons Attribution-NonCommercial-NoDerivs 4.0 Unported License. Permissions beyond the scope of this license may be available at www.revistamedicocientifica.org. 


\section{INTRODUCCIÓN}

La gastroenteritis aguda (GEA) es una de las principales causas de morbilidad y mortalidad en la infancia. En los países en vías de desarrollo se producen cada año de 3 a 5 millones de muertes en niños menores de 5 años. ${ }^{1}$ En el curso de una GEA puede producirse un desequilibrio entre los ingresos y las pérdidas de agua libre o de sodio sérico y originarse una hiponatremia, es decir, la presencia de sodio sérico por debajo de $135 \mathrm{mEq} / \mathrm{L}$. Según la gravedad, se puede clasificar en leve (concentraciones de sodio sérico entre 134 - $130 \mathrm{mEq} / \mathrm{L}$ ); moderada (entre 129 - $125 \mathrm{mEq} / \mathrm{L}$ ); y severa, (sodio sérico por debajo de $125 \mathrm{mEq} / \mathrm{L}$ ), aunque también es muy conveniente valorar el estado de hidratación del paciente. ${ }^{2,3}$

Numerosos estudios han confirmado la seguridad y eficacia de la terapia de rehidratación oral, por lo que se considera el método preferido de rehidratación en niños con GEA. Sin embargo, la rehidratación intravenosa se continúa utilizando en escenarios donde no es posible hidratar al paciente por vía enteral (niños con deterioro neurológico, vómitos incoercibles y deshidratación grave). ${ }^{4}$

La administración de líquidos hipotónicos intravenosos se basa en trabajos realizados en la década de 1950, que describe los requerimientos de líquidos y electrolitos en niños sanos en función del gasto energético y composición de la leche. Por lo general, los líquidos de mantenimiento intravenosos (IV) hipotónicos han seguido siendo el estándar de atención en la mayoría de las instituciones de salud. Durante la última década se han acumulado pruebas que sugieren que la administración de un líquido de mantenimiento IV hipotónico en pacientes críticamente enfermos, aumenta el riesgo de hiponatremia y la misma puede producir convulsiones, deterioro neurológico y muerte. $^{5}$

En los últimos años, muchos estudios e informes de casos han demostrado que los fluidos hipotónicos que contienen entre $30.8-74 \mathrm{mEq} / \mathrm{L}$ de sodio, pueden conducir a una hiponatremia grave y ocasionalmente pueden causar daño neurológico permanente o la muerte. ${ }^{6}$ En el año 2016 egresaron de la salas de Pediatría del Hospital del Niño José Renán Esquivel, un total de 1108 pacientes con diagnósticos de gastroenteritis y deshidratación, a los que se practicaron colocaciones de sueros hipotónicos durante su estancia hospitalaria. ${ }^{7}$

El propósito del estudio es determinar la frecuencia de hiponatremia en pacientes con gastroenteritis que reciben soluciones salinas hipotónicas en salas de Pediatría del Hospital del Niño Dr. José Renán Esquivel entre enero a diciembre del 2016.

La correcta detección de las variables es fundamental para la elaboración de registros estadísticos actualizados, la revisión de normas de manejo actuales, el establecimiento de nuevos protocolos de manejo específicos a la unidad y adición de esta información a la literatura científica. ${ }^{8-10}$ Además de proveer bases estadísticas para definir protocolos de atención actualizadas en las salas de pediatría del Hospital del Niño Dr. José Renán Esquivel, el estudio servirá de marcador inicial para la prevención de hiponatremia en pacientes deshidratados hospitalizados con gastroenteritis y establecerá un modelo para la realización de estudios similares en otros centros Pediátricos de Panamá permitiendo así, la comparación interinstitucional de datos.

\section{MATERIALES Y MÉTODOS}

\section{TIPO DE ESTUDIO}

Realizaremos un estudio de corte transversal descriptivo retrospectivo. Examinaremos los expedientes clínicos de 190 niños sin antecedentes patológicos previos de 1 mes a 15 años, que habían ingresado a sala de pediatría con gastroenteritis aguda y deshidratación entre enero a diciembre de 2016.

Área Problema: Salas de Pediatría del Hospital del Niño Dr. José Renán Esquivel de enero a diciembre de 2016

Área Temática: Hidratación en pacientes hospitalizados con gastroenteritis con soluciones salinas hipotónicas.

\section{POBLACIÓN Y DISEÑO MUESTRAL}

Examinaremos los expedientes clínicos de 190 niños con diagnóstico de gastroenteritis aguda sin antecedentes personales patológicos de 1 mes a 15 años, estos habían ingresado a salas de Pediatría del Hospital del Niño Dr. José Renán Esquivel entre enero a diciembre de 2016.

\section{TAMAÑO MUESTRAL}

Para la obtención de la muestra utilizamos un 95\% de confianza, la muestra obtenida fue de 190 pacientes y fue calculada utilizando el programa Epi Info versión 7 


\section{CRITERIOS DE INCLUSIÓN}

- Todo paciente con cuadro de gastroenteritis que tengan niveles de sodio sérico comprendidos entre $135 \mathrm{mEq} / \mathrm{L}$ $145 \mathrm{mEq} / \mathrm{L}$.

- Todo paciente de 1 mes hasta 15 años que ingresa a salas de Pediatría del Hospital del Niño Dr. José Renán Esquivel con gastroenteritis y deshidratación en el periodo de estudio.

- Pacientes que recibieron hidratación desde su ingreso a sala con venoclisis de D/SS $0.33 \%$ o D/SS $0.45 \%$.

- Pacientes que tenían al menos dos mediciones de sodio sérico (uno al ingreso y el segundo entre $6 \mathrm{~h}$ y $24 \mathrm{~h}$ después de iniciado el uso de soluciones hipotónicas).

- Para niños con hospitalizaciones múltiples, solo se consideró la primera hospitalización.

\section{CRITERIOS DE EXCLUSIÓN}

- Pacientes con antecedentes de nefropatías, cardiopatías, enfermedades crónicas, patología hipotálamo- hipofisaria y trastornos neurológicos.

- Pacientes con patología quirúrgica reciente.

- Pacientes procedentes de sala de terapia intensiva debido a la gravedad del proceso.

- Pacientes que utilizaron diuréticos.

Dentro de una muestra total de 1150 pacientes se revisaron 766 expedientes al azar hasta completar la muestra estudio de 190 casos que cumplían con los criterios de inclusión y exclusión.

\section{PROCEDIMIENTO PARA LA RECOLECCIÓN DE DATOS}

Se elaboró un instrumento de recolección de datos que contiene las variables a estudiar, sin datos que identifiquen al paciente.

Previa aprobación del Departamento de Docencia Médica y el Comité de Bioética, se solicitó a Registros y Estadísticas de Salud del Hospital del Niño Dr. José Renán Esquivel, el listado de pacientes hospitalizados en el periodo de enero del 2016 a diciembre del 2016 con diagnóstico de gastroenteritis aguda. Posteriormente, se hizo la solicitud de los expedientes clínicos al departamento de Archivos Clínicos para su análisis.

\section{PLAN DE ANÁLISIS DE RESULTADOS}

Una vez recolectada la información de los expedientes clínicos, creamos una base de datos en Excel versión 16.14.1, la cual fue depurada y exportada al programa estadístico Epi Info versión 7.0.
Realizamos un análisis descriptivo univariado con frecuencias y proporciones para las variables categóricas. Para las variables que siguen una distribución normal se utilizó la mediana.

\section{RESULTADOS}

Del universo de 1150 pacientes admitidos entre enero de 2016 a diciembre 2016 con diagnóstico de gastroenteritis aguda, ingresaron al estudio 190 pacientes que cumplieron con criterios de inclusión y exclusión.

Se registraron mayor frecuencia de pacientes masculinos (60\%) con diagnóstico de gastroenteritis en relación con el sexo femenino. (Ver Tabla 1).

Tabla 1. Distribución según sexo de los pacientes con diagnóstico de gastroenteritis aguda tratada con líquidos intravenosos.

\begin{tabular}{lll}
\hline SEXO & FRECUENCIA & PORCENTAJE (\%) \\
\hline FEMENINO & 76 & 40 \\
MASCULINO & 114 & 60 \\
TOTAL & 190 & 100 \\
\hline
\end{tabular}

Fuente: Registros y Estadísticas de Salud. Hospital del Niño Dr. José Renán Esquivel. Ciudad de Panamá. 2016

El 89.5\% fueron menores de 5 años, 35.8\% lactantes menores, $29.5 \%$ lactantes mayores, $24.2 \%$ preescolares, $8.9 \%$ escolares y $1.6 \%$ adolescentes. (Ver Tabla 2).

Tabla 2. Distribución según grupo etario de los pacientes con diagnóstico de gastroenteritis aguda tratada con líquidos intravenosos.

\begin{tabular}{lll}
\hline GRUPO ETARIO & FRECUENCIA & PORCENTAJE (\%) \\
\hline LACTANTE MENOR & 68 & 35.8 \\
LACTANTE MAYOR & 56 & 29.5 \\
PRE-ESCOLAR & 46 & 24.2 \\
ESCOLAR & 17 & 8.9 \\
ADOLESCENTE & 3 & 1.6 \\
TOTAL & 190 & 100 \\
\hline
\end{tabular}

Fuente: Registros y Estadísticas de Salud. Hospital del Niño Dr. José Renán Esquivel. Ciudad de Panamá. 2016

La mediana del sodio de ingreso fue $137 \mathrm{mEq} / \mathrm{L}$ (RIC: 135-145 $\mathrm{mEq} / \mathrm{L})$. El $25 \%$ no recibió cargas de cristaloides y el $61 \%$ recibió sólo 1 carga. Para la hidratación intravenosa de pacientes admitidos en nuestra institución con gastroenteritis aguda se utilizaron 2 tipos de soluciones hipotónicas; la D/SS $0.33 \%$ fue 118 (62.1\%) y D/SS $0.45 \%$ fue 72 (37.9\%), luego de su ingreso. En la mayoría de los pacientes (92.6\%), el estado mental al momento del ingreso fue alerta. 
Basándonos en el llenado capilar >2 segundos como signo clínico de hipoperfusión, el $15.8 \%$ de los pacientes presentaban estado de choque.

Sólo 42 pacientes del estudio (22\%) tenían consignada la presión arterial en su historia de admisión, de estos el $26.2 \%$ presentaban choque no compensado

Solo se evaluó la gravedad específica a 43 pacientes del estudio, de los cuales $34.9 \%$ presentaban datos de deshidratación. (Ver Tabla 3)

Tabla 3. Características clínicas al momento del ingreso en los pacientes con diagnóstico de gastroenteritis aguda tratada con líquidos intravenosos.

\begin{tabular}{cc}
\hline VARIABLE & VALOR \\
\hline NÚMERO DE CARGAS DE CRISTALOIDES & $\mathrm{n}(\%)$ \\
Ninguna & $49(25.7)$ \\
1 & $116(61.1)$ \\
2 & $23(12.1)$ \\
3 & $2(1.1)$ \\
TIPO DE SOLUCIÓN & $\mathrm{n}(\%)$ \\
D/SS 0.33\% & $118(62.1)$ \\
D/SS 0.45\% & $72(37.9)$ \\
Alerta & $\mathrm{n}(\%)$ \\
Irritable & $176(92.6)$ \\
Letárgico & $8(4.2)$ \\
Si & $6(3.2)$ \\
No & $\mathrm{n}(\%)$ \\
CHOQUE & $30(15.8)$ \\
PRESIÓN ARTERIAL (42) & $160(84.2)$ \\
Hipotenso & $\mathrm{n}(\%)$ \\
Normotenso & $11(26.2)$ \\
>1.025 & $31(73.8)$ \\
S1.025 & $\mathrm{n}(\%)$ \\
\hline GRAVEDAD ESPECÍFICA (43) & $15(34.9)$ \\
\hline
\end{tabular}

Fuente: Registros y Estadísticas de Salud. Hospital del Niño Dr. José Renán Esquivel. Ciudad de Panamá. 2016

Luego de 6 a $24 \mathrm{~h}$ posterior a su ingreso, la media de sodio sérico fue de 136.6 +/- $2.5 \mathrm{mEq} / \mathrm{L}$. El $24 \%$ no presentó evacuaciones posteriores a su ingreso y el $45.3 \%$ presentó entre 1 y 3 evacuaciones. La mayoría de los pacientes (79.5\%) no tuvieron vómitos durante las primeras $24 \mathrm{~h}$ de hospitalización. La media de la estancia hospitalaria fue de 2 días con un rango entre 1 y 25 días (Tabla 4).
Tabla 4. Características clínicas en las primeras $24 \mathrm{~h}$ luego de su ingreso en los pacientes con diagnóstico de gastroenteritis aguda tratada con líquidos intravenosos.

\begin{tabular}{lc}
\hline VARIABLE & VALOR \\
\hline Na LUEGO DE 6 - 24 H DEL INGRESO & Mediana (RIC) \\
NÚMERO DE EVACUACIONES & $137(130-144)$ \\
Ninguna & $\mathrm{n}(\%)$ \\
$1-3$ & $46(24.2)$ \\
$4-7$ & $86(45.3)$ \\
$\geq 8$ & $45(23.7)$ \\
NÚMERO DE VÓMITOS & $13(6.8)$ \\
Ninguno & $\mathrm{n}(\%)$ \\
$4-3$ & $151(79.5)$ \\
$4-7$ & $33(17.4)$ \\
ESTANCIA INTRAHOSPITALARIA & $6(3.2)$ \\
& Mediana (RIC) \\
\hline
\end{tabular}

Fuente: Registros y Estadísticas de Salud. Hospital del Niño Dr. José Renán Esquivel. Ciudad de Panamá. 2016

Al comparar la media de los niveles de sodio luego de las 6 y 24 $\mathrm{h}$ del ingreso, utilizando ambas soluciones hipotónicas, no hubo diferencias significativas para el desarrollo de hiponatremia.

Tampoco hubo diferencias significativas en cuanto a la estancia hospitalaria de acuerdo con el tipo de solución salina hipotónica utilizada. (Ver Tabla 5)

Tabla 5. Comparación según tipo de solución hipotónica recibida en los pacientes con diagnóstico de gastroenteritis aguda tratada con líquidos intravenosos.

\begin{tabular}{llll}
\hline VARIABLE & D/SS 0.33\% & D/SS 0.45\% & P \\
\hline $\begin{array}{l}\text { Na LUEGO DE 6 - 24 H } \\
\text { DEL INGRESO }\end{array}$ & Mediana & Mediana & \\
& (RIC) & (RIC) & $0.69 *$ \\
ESTANCIA & $137(130-144)$ & $136(130-143)$ & \\
HOSPITALARIA & Media (RIC) & Mediana & \\
& $2(1-25)$ & (RIC) & $0.39 *$ \\
& & $2(1-14)$ & \\
\hline
\end{tabular}

* Comparación de medianas con la Prueba de Mann-Whitney.

Fuente: Registros y Estadísticas de Salud. Hospital del Niño Dr. José Renán Esquivel. Ciudad de Panamá. 2016

En cuanto a las complicaciones, solo se registró un paciente lactante mayor que ingresa con valores de sodio en rango normales, que durante sus primeras $24 \mathrm{~h}$ intrahospitalaria presenta episodio convulsivo no asociado a fiebre ni a alteración hidroelectrolítica. Fue evaluado por el servicio de Neurología que encuentra neurodesarrollo y neuroexamen 
normales, sin datos de riesgo perinatal ni familiares con epilepsia. Coprocultivo negativo por bacterias.

\section{DISCUSIÓN}

En nuestro estudio se encontró una mayor frecuencia de pacientes con gastroenteritis aguda en el sexo masculino 114 (60\%), datos similares a los publicados en España por Sánchez M y grupo en un estudio con 205 casos cuya frecuencia en niños fue de 117 (57\%). ${ }^{2}$ Otro estudio publicado de 180 casos en nuestro país por Luciani K y colaboradores en el año 2012, demuestra que en pacientes con gastroenteritis aguda la frecuencia para el sexo masculino fue de 109 (60.6\%) respecto al femenino $71(39.4 \%){ }^{25}$

El $89.5 \%$ de los pacientes admitidos con gastroenteritis a nuestra institución fueron menores de 5 años, datos muy similares a los obtenidos por Surribas $M$ y colaboradores, quienes evaluaron 307 pacientes pediátricos encontrando una frecuencia de $247(80,4 \%) .{ }^{26}$ En un estudio local de 180 casos se encontró una frecuencia de 169 (93.9\%) de casos en niños menores de 5 años. ${ }^{25}$

El 25\% de nuestros pacientes no recibió cargas de cristaloides y el $61 \%$ recibió sólo 1 carga. En un estudio publicado en el año 2010 en la Revista de Nefrología Pediátrica, se evaluaron 124 niños con gastroenteritis aguda que ingresaron con sodio sérico normal, todos los pacientes fueron tratados con líquidos hipotónicos y de estos 100 habían recibido un bolo de solución salina inicial de $21.05 \pm 8.5 \mathrm{ml} / \mathrm{kg}$ al ingreso. A pesar de esto el nivel sérico de $\mathrm{Na}$ disminuyó en $1.7 \pm 4.3 \mathrm{mEq} / \mathrm{L}$ en todo el grupo, indistintamente de los bolos utilizados. ${ }^{27}$

Basándonos en el llenado capilar $>2$ segundos como signo clínico de hipoperfusión, el $15.8 \%$ de los pacientes presentaban estado de choque, de los cuales ninguno se consignó con este diagnóstico a su ingreso. Existen diversos programas educativos de reanimación pediátrica que establecen que un tiempo de llenado capilar $>2$ segundos es un buen indicador de choque como lo respalda la publicación de Bústos $\mathrm{R}$ y Padilla $\mathrm{O}$, en el año $2014 .^{28}$ Otra publicación presentada en la revista Pediatrics en el año 2006 encontraron dentro de un total de 83 niños (menores de 5 años), con gastroenteritis aguda que llegaban a urgencias de un hospital de tercer nivel de complejidad que 13 de ellos (15.6\%) presentaban signos de choque (Ilenado capilar $>2$ segundos)..$^{29}$

Sólo 42 pacientes del estudio (22\%) tenían consignada la presión arterial en su historia de admisión, de estos el $26.2 \%$ presentaban choque no compensado.
Se realizó un estudio entre 2013 - 2016 en Kenya. Se evaluaron 42,937 niños que ingresaron con edad comprendida entre 1 mes y 5 años, sin comorbilidades, cirugías previas, ni quemaduras. La prevalencia del choque diagnosticado clínicamente fue del 1,5\% (622 casos). Los casos identificados consistían en choque hipovolémico secundario a deshidratación / diarrea 582/622 (94\%), con una alta mortalidad 211/622 (34\%). ${ }^{30}$

Solo se evaluó la gravedad específica a 43 pacientes del estudio, de los cuales $34.9 \%$ presentaban datos de deshidratación a pesar de que existe literatura científica que avala el uso de la gravedad específica para determinar el grado de hidratación de un paciente. ${ }^{14}$ Se reportó un estudio de cohortes prospectivo en el Departamento de Emergencia del Childrens Hospital de Los Ángeles, donde 79 sujetos con edades entre 3 meses y 36 meses con gastroenteritis, en la cual se sospechó de deshidratación moderada, clínicamente se le evaluó la gravedad específica. El mismo concluye que los índices urinarios no son pruebas diagnósticas útiles para identificar la presencia de deshidratación durante la evaluación inicial de los niños con gastroenteritis. ${ }^{12}$

Aunque la asociación entre la administración de líquido hipotónicos y el aumento de las tasas de hiponatremia se ha descrito previamente, en nuestro estudio luego de 6-24 h posterior a su ingreso, la media de sodio sérico fue de 136.6 $\pm 2.5 \mathrm{mEq} / \mathrm{L}$. En una revisión sistemática publicada en octubre de 2017 se identificaron 603 artículos, de los cuales se incluyeron 6 ( 3 ensayos controlados aleatorizados y 3 estudios observacionales). Los resultados de estudios únicos demostraron que en 24 h, la administración de solución salina isotónica no se asoció con una disminución significativa del sodio sérico, mientras que las soluciones hipotónicas $(0,2$ $0,45 \%$ solución salina) se asociaron en un estudio, con descensos séricos de sodio de 1,3 mEq/L (139.2, DS 2.9 - 137.9, DS 2.5) en 133 niños pequeños (de entre 1-28 meses), a 5.7 $\mathrm{mEq} / \mathrm{L}$ (DS 3.1) en un subgrupo de 18 niños mayores (edad media 5.8, DS 2.7 años). ${ }^{4}$ En otro meta-análisis publicado en la Revista Pediatrics, se realizaron búsquedas en PubMed, Embase, Biblioteca de Cochrane y clinicaltrials.gov donde se incluyeron 10 estudios controlados aleatorizados. El metanálisis mostró un riesgo significativamente mayor de fluidos hipotónicos intravenosos (D/SS $0.18 \%$, D/SS $0.33 \%$ y D/SS $0.45 \%$ ) para desarrollar hiponatremia (RR 2.24, IC 95\% 1.52 a 3.31) e hiponatremia grave (RR 5.29, IC 95\% 1.74 a 16.06). Hubo una disminución significativamente mayor de sodio sérico en los niños que recibieron líquidos intravenosos hipotónicos ($3.49 \mathrm{mmol} / \mathrm{L}$ versus líquidos IV isotónicos, IC del 95\%: -5.63 a 1.35). No se encontraron diferencias significativas entre las 2 
intervenciones en el riesgo de hipernatremia (RR 0.73; IC del 95\%: 0.22 a 2.48$)^{24}$

El 24\% no presentó evacuaciones posteriores a su ingreso hospitalario y el $45.3 \%$ presentó entre 1 y 3 evacuaciones. La mayoría de los pacientes (79.5\%) no tuvieron vómitos durante las primeras $24 \mathrm{~h}$ de hospitalización. A pesar de que ambas vías se puede perder sodio en pacientes que presentan gastroenteritis, en la literatura revisada no fue tomado en cuenta dichas condiciones para el análisis de sus datos.

La mediana de la estancia hospitalaria fue de 2 días con un rango entre 1 y 25 días. En un estudio realizado en el 2008 en nuestro país, reporta un promedio de estancia en el hospital de 2.9 días que generaba un costo de hospitalización por paciente de 153.7 dólares. ${ }^{31}$ En otro estudio prospectivo observacional de pacientes de 1 mes a 14 años que ingresaron al Hospital de Palamós, Girona - España, la estancia media fue de 3.1 días y tan solo 3 (4.6\%) tuvieron una hospitalización igual o superior a 7 días. ${ }^{32}$

No hubo diferencias significativas en cuanto a la estancia hospitalaria de acuerdo con el tipo de solución salina hipotónica utilizada. En cuanto a las complicaciones solo se registró un paciente lactante mayor que ingresa con valores de sodio en rango normales, pero durante sus primeras 24 horas intrahospitalaria presenta episodio convulsivo no asociado a fiebre, ni a alteración hidroelectrolítica. Fue evaluado por el servicio de Neurología que encuentra neurodesarrollo y neuroexamen normales sin datos de riesgo perinatal ni familiares con epilepsia. Coprocultivo negativo por bacterias. En un metanálisis publicado en el 2014 el cual evalúa la evidencia de los ensayos controlados aleatorizados sobre la seguridad de los líquidos de mantenimiento intravenosos isotónicos frente a los hipotónicos en los niños hospitalizados, se informaron resultados adversos de interés en 2 estudios, ambos atribuidos al uso de soluciones hipotónicas (D/SS 0.33\%). Kannan y cols informaron 1 caso de encefalopatía hiponatremia con convulsiones y estupor en un niño que recibió líquido hipotónico. Choong et al informaron convulsiones de novo en 1.54\% (2 de 130) de los niños que recibieron líquido hipotónico y ninguno en los receptores de fluido isotónico. La duración de la hospitalización fue similar entre los 2 tipos de líquidos (Isotónicos vs Hipotónicos). ${ }^{17}$

\section{CONCLUSIÓN}

En el estudio se encontró mayor frecuencia de pacientes de sexo masculino, no se registró hiponatremia en pacientes con gastroenteritis aguda que reciben soluciones salinas hipotónicas en las salas de Pediatría del Hospital del Niño Dr. José Renán Esquivel.

Para la hidratación intravenosa de pacientes admitidos en nuestra institución con gastroenteritis aguda, se utilizaron 2 tipos de soluciones hipotónicas: la D/SS 0.33\% fue 118 (62.1\%) y D/SS $0.45 \%$ fue 72 (37.9\%). Al comparar la mediana de los niveles de sodio luego de las 6 y $24 \mathrm{~h}$ del ingreso, utilizando ambas soluciones hipotónicas, no hubo diferencias significativas para el desarrollo de hiponatremia. Basándonos en el llenado capilar >2 segundos como signo clínico de hipoperfusión, el $15.8 \%$ de los pacientes presentaban estado de choque. Dentro del cual, a pesar de que solo 42 casos presentaban toma de presión arterial al momento de su ingreso, de estos el $26.2 \%$ presentaban choque no compensado.

\section{RECOMENDACIONES}

Perder el temor que ha existido durante décadas a las complicaciones secundarias del uso de soluciones con mayor concentración de sodio al momento de administrar líquidos intravenosos.

\section{RECONOCIMIENTOS}

Dr. Florencio McCarthy y Dr. Luis Coronado por su colaboración de literatura y revisión de nuestro estudio.

A la Lic. Vilma y todo el personal de estadísticas por su ayuda invaluable en la recolección de datos.

\section{REFERENCIAS}

1. Aguilar F, Lasso C.E, Vázquez F, Fernández $F$, et al. Gastroenteritis aguda en la infancia. Medicina de urgencias y emergencias. 5ta Edición, año 2015 Elsevier España. Capítulo 180, 913-917.

2. Sánchez M, Martín R, Cano J, Villalobos E. Fluid therapy and iatrogenic hyponatraemia risk in children hospitalised with acute gastroenteritis: prospective study. Revista Nefrología 2014;34(4):477-82.

3. Medrano A, Ortega R, Torres E, Macario A, Barrera J. Hiponatremia y su etiología en pacientes pediátricos ingresados a sala de urgencias. Rev Med Inst Mex Seguro Soc. 2017;55 Supl $1:$ S64-70 
4. Grisaru S, Xie J, Samuel S, Freedman S. Iatrogenic Dysnatremias in Children with Acute Gastroenteritis in HighIncome Countries: A Systematic Review. Front. Pediatr. 5:210. doi:10.3389/fped.2017.00210

5. Friedman J, Beck C, DeGroot J, et all. Comparison of Isotonic and Hypotonic Intravenous Maintenance Fluids Randomized Clinical Trial. JAMA Pediatr. 2015; 169(5):445-451. doi:10.1001/jamapediatrics.2014.3809

6. Ouchi K, Sugiyama K. Hypotonic fluid reduce serum sodium compared to isotonic fluids during anesthesia induction in pediatric patients undergoing maxillofacial surgery-type of infusion affects blood electrolytes and glucose: an observational study. BMC Pediatrics (2016) 16:112 DOI 10.1186/s12887-016-0650-6

7. Registros y estadísticas de salud del Hospital del Niño Dr. José Renán Esquivel, año 2016.

8. Normas de manejo de problemas pediátricos. Departamento de docencia. Patronato del Hospital del Niño. 4ta re-edición 2016. Titulo 1: pág 82-95.

9. Suárez L, Cano B. Current management of acute gastroenteritis (AGE) with oral rehydration solutions. Nutr. clín. diet. hosp. 2009; 29(2):6-14

10. Iro M, Sell T, Brown N, Maitland K, Rapid intravenous rehydration of children with acute gastroenteritis and dehydration: a systematic review and meta-analysis. BMC Pediatrics. DOI 10.1186/s12887-018-1006-1

11. Contraloría General de la República. Dirección de Estadística y Censo. Portal de Estadística Panameña. Ministerio de Salud, Departamento de Análisis y Tendencias de Salud, REMES. Año 2014

12. Steiner MJ, Nager AL, Wang VJ: Urine specific gravity and other urinary indices: inaccurate test for dehydration, Pediatr Emerg Care, 2007, 23:298-303

13. Bonadio WA, Hennes $\mathrm{HH}$, Machi J, Madagame E: Efficacy of measuring BUN in assessing children with dehydration due to gastroenteritis, Ann Emerg Med, 1989, 18:755-757.

14. Marcdante K, Kliegman R. Dehydration and Replacement Therapy Nelson Essentials of Pediatrics, Eighth Edition. Chapter 33, 126-130

15. McNab S, Isotonic vs Hypotonic Intravenous Fluids for Hospitalized Children. JAMA August 18, 2015 Volume 314, Number 7. 720-721
16. Foster B, Tom D, Hill V. Hypotonic versus Isotonic Fluids in Hospitalized Children: A Systematic Review and MetaAnalysis. J Pediatr 2014;165: 163-9). doi.org/10.1016/j.jpeds.2014.01.040

17. Raksha S, Dakshayani B, Premalatha R. Full Volume Isotonic (0.9\%) vs. Two-Thirds Volume Hypotonic (0.18\%) Intravenous Maintenance Fluids in Preventing Hyponatremia in Children Admitted to Pediatric Intensive Care Unit-A Randomized Controlled Study. Journal of Tropical Pediatrics, 2017, 0, 1-7 doi:10.1093/tropej/fmx012

18. Carandang F, Anglemyer A, Longhurst C, et all. Association Between Maintenance Fluid Tonicity and Hospital Acquired Hyponatremia. J Pediatr. 2013 December; 163(6). doi:10.1016/j.jpeds.2013.07.020.

19. Tran $M$, Tantsis E, Ging J. In children requiring intravenous fluid for hydration maintenance, which out of hypotonic saline and isotonic saline is less likely to result in the development of hyponatraemia?. Journal of Pediatrics and Child Health 53 (2017). Pág: 309-313.doi:10.1111/jpc.13265

20. Neville K, Sandeman D, Rubinstein A, McGlynn M, Walker J. Prevention of Hyponatremia during Maintenance Intravenous Fluid Administration: A Prospective Randomized Study of Fluid Type versus Fluid Rate. J Pediatr 2010;156:313-9. Vol. 156, No. 2. 10.1016/j.jpeds.2009.07.059

21. McNab S, Ware RS, Neville KA, Choong K, Coulthard MG, Duke T, Davidson A, Dorofaeff T. Isotonic versus hypotonic solutions for maintenance intravenous fluid administration in children (Review). The Cochrane Library 2014, Issue 12

22. Shukla S, Basu S, Moritz M. Use of hypotonic Maintenance intravenous Fluids and hospital- acquired hyponatremia remain common in children admitted to a general Pediatric Ward. Front. Pediatr. 4:90. doi:10.3389/fped.2016.00090

23. Toledo J, Morell C, Vento M. Intravenous isotonic fluids induced a positive trend in natraemia in children admitted to a general pediatric ward. Acta Pædiatrica. Volume 105, Issue 6. June 2016, Pages e263-e268. DOI:10.1111/apa.13316

24. Wang J, Xu E, Xiao Y. Isotonic Versus Hypotonic Maintenance IV Fluids in Hospitalized Children: A MetaAnalysis. Pediatrics 2014;133:105-113. doi:10.1542/peds.2013-2041

25. Luciani K, Estripeaut D, Cisterna O, et all. Gastroenteritis viral en la era post - introducción de la vacuna contra el rotavirus. Pediatr Panamá 2012; 41(1):6-11 
26. Surribas $M$, López $M$, Valverde $M$, et all. Epidemiología de la gastroenteritis aguda en el servicio de pediatría del h. San pedro de alcántara (cáceres). Vox paediatrica.2007, Volumen 15. No1.

27. Hanna $M$, Saberi $M$. Incidence of hyponatremia in children with gastroenteritis treated with hypotonic intravenous fluids. Pediatr Nephrol (2010) 25:1471-1475

28. Bustos R, Padilla O. El tiempo de llene capilar prolongado es predictor de una saturación venosa central de oxígeno disminuida. Rev Chil Pediatr 2014; 85 (5): 539-545

29. Shavit I, Brant R, Nijssen-Jordan C, et all. A Novel Imaging Technique to Measure Capillary- Refill Time: Improving Diagnostic Accuracy for Dehydration in Young Children with Gastroenteritis. PEDIATRICS (ISSN Numbers: Print, 0031-4005;

30. Mbevi G, Ayieko P, Irimu G, Akech S, English M. Prevalence, a etiology, treatment and outcomes of shock in children admitted to Kenyan hospitals. Mbevi et al. BMC Medicine (2016) 14:184.

31. Constenla D, Ortega E, Rheingansa R, Antila L, SáezLlorens $X$. Impacto económico de la vacuna antirrotavirus en Panamá. An Pediatr (Barc). 2008;68(2):128-35.

32. Parada E, Inoriza J, Plaja P. Gastroenteritis aguda: coste de una causa de ingreso potencialmente evitable. An Pediatr (Barc). 2007;67(4):368-73 